\title{
Identification of adipogenesis specific microRNAs
}

\author{
ML. Cherradi 1 , N. Perwitz, R. Werner 2 , G. Brabant 1 \\ 1 Department of Internal Medicine I, University of Luebeck, Germany;

\section{Introduction}

Obesity is grave and rising problem in
Western societies. This disease shows a large
degree of depot- and sex-specific variation
and an understanding of the regulatory
mechanisms underlying this variation is
crucial to identify potential therapeutic
targets or treatment strategies to combat
obesity. MicroRNAs are recently recognized
important players in adipogenesis and fat
metabolism [1]. They posttranscriptionally
regulate diverse biological processes, e.g. the
proliferation and differentiation of cells.
Here we studied the fat depot and sex
hormone-specific regulation of microRNAs
during differentiation.

Results

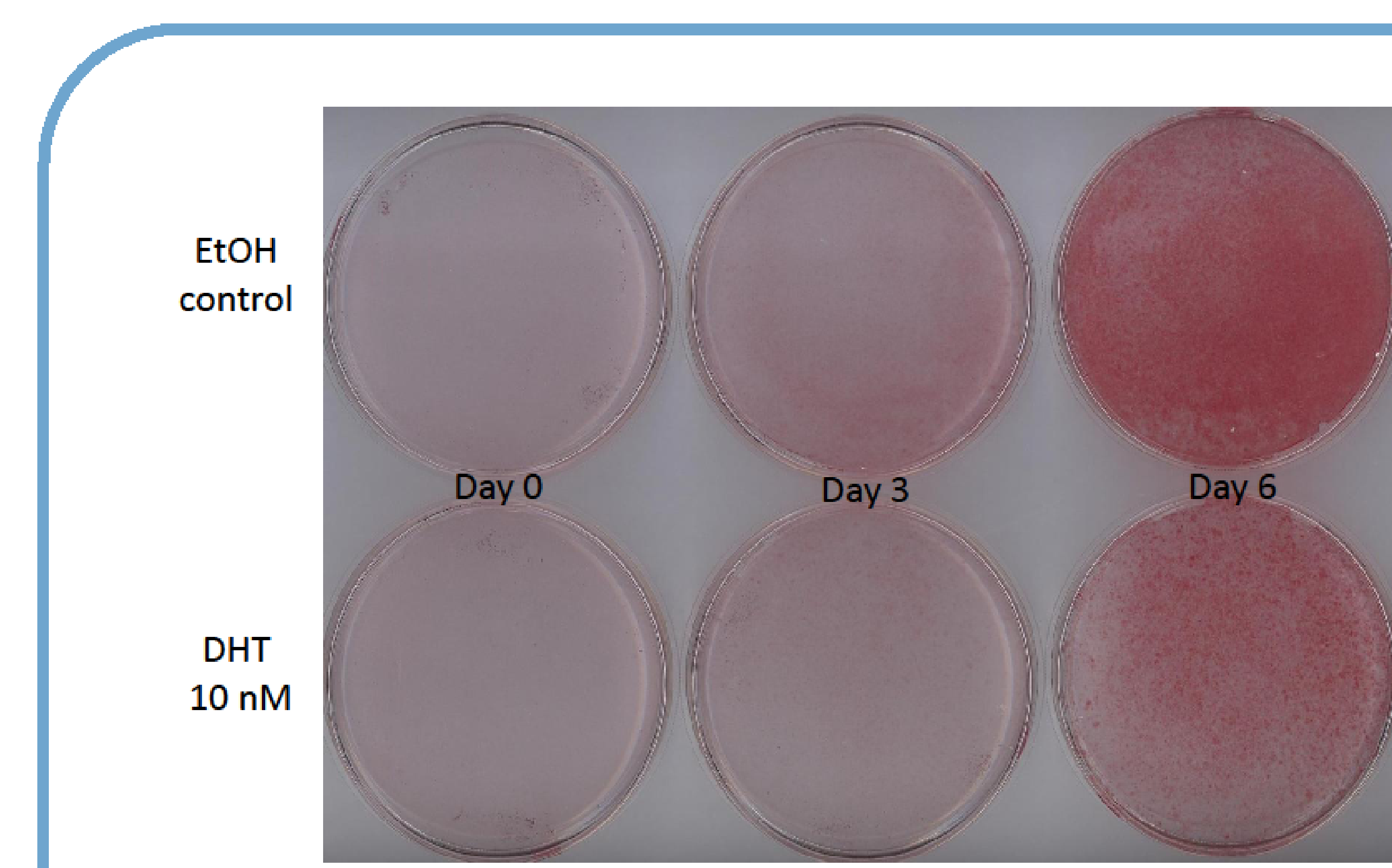

DHT attenuates lipid accumulation in epiWAT.

Oil Red O staining reveals less lipid content in DHT stimulated cells compared to controls (EtOH) at days 3 and 6 of fat cell differentiation, indicating a decelerated differentiation to mature adipocytes.
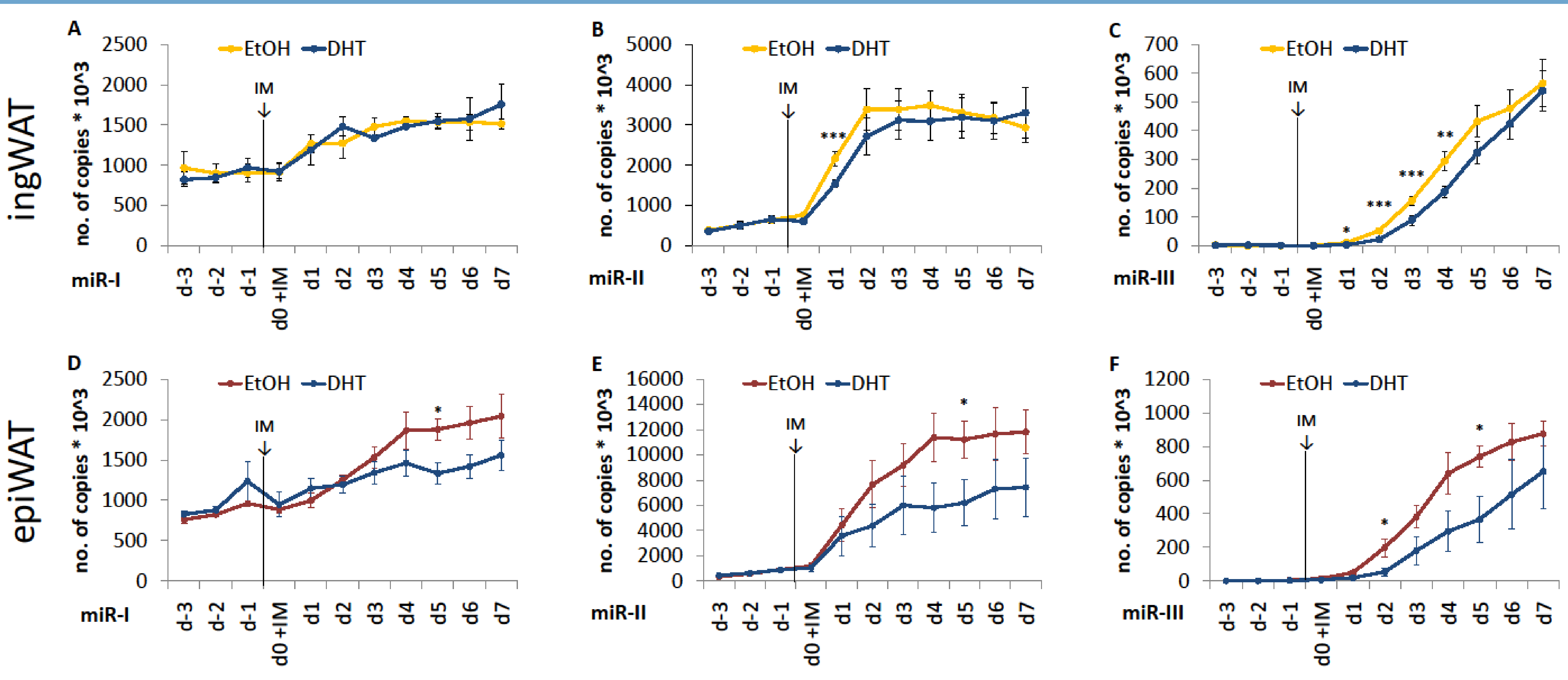

DHT regulates microRNA expression during adipocyte differentiation.

MicroRNA expression profiles of microRNAs miR-I (A,D), miR-II (B,E) and miR-III (C,F) in ingWAT (A-C, yellow) and epiWAT (D-F, red) cell lines in control conditions (EtOH) and chronically stimulated with DHT (blue). DHT (significantly) decreases the expression of miR-II and miR-III, indicating a regulation of these microRNAs during differentiation. All datasets were normalized to miR-191 (housekeeping).

$$
\begin{aligned}
& \mathrm{N} \\
& \text { di } \\
& \text { e) } \\
& \text { b } \\
& \text { w } \\
& \text { d }
\end{aligned}
$$

MicroRNAs are
differently
expressed
between
white fat
depots.
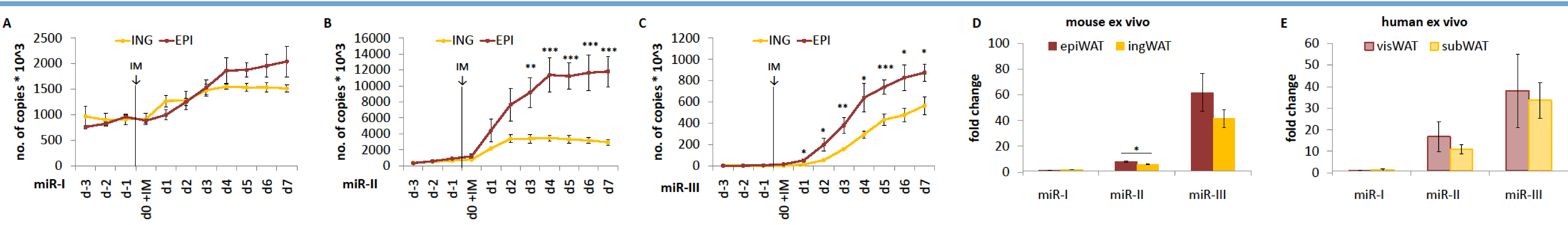

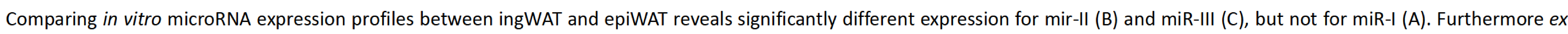

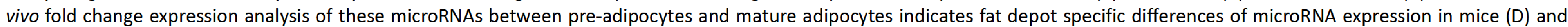
humans (E). Fold change was calculated by $2^{\wedge} \Delta \Delta C$ t method. All datasets were normalized to miR- 191 .

\section{Summary \& Conclusion}

Stimulation with sex-hormone DHT.

.. decelerates epiWAT adipocyte differentiation

uncovers microRNA regulation during adipogenesis

$\checkmark$ MicroRNA expression varies (significantly) between ingWAT and epiWAT fat depots in vitro and ex vivo in mice and humans

$>$ MicroRNAs are specifically expressed in adipocyte differentiation and their expression can be influenced by sexhormones and fat depot specific characteristics.

\section{Literature}

[1] P. Arner and A. Kulyté MicroRNA regulatory networks in human adipose tissue and obesity. Nat Rev Endocrinol. 2015 May;11(5):276-88

\section{Material \& Methods}

Cell culture: Inguinal (ing) and epididymal (epi) white adipose tissue (WAT) SV-40 immortalized pre-adipocytes were seeded in differentiation medium (DM). 24 hours later chronic stimulation with $10 \mathrm{nM}$ male sex-hormone dihydrotestosterone (DHT) or EtOH (control) started. Confluent cells were induced for 24 hours with induction medium (IM = DM + IBMX, indomethacine, dexamethasone). Cells maintained in DM until they reached mature adipocyte phenotype Mice \& Humans: IngWAT and epiWAT from C57BL/6 mice and subcutaneous and visceral WAT from men were ex vivo digested with collagenase $A$; pre-adipocytes and mature adipocytes were separated by centrifugation. $R T-q P C R$ : Universal cDNA Synthesis Kit II, miRCURY LNA ${ }^{\mathrm{TM}}$ RT PCR primer sets and ExiLENT SYBR ${ }^{\circledast}$ miRCURY LNA ${ }^{\text {TM }}$ microRNA PCR (Exiqon) were used to the manufactures instructions and recommendations. Data sets were normalized to miR-191-5p. Western Blot: Equal concentrations of protein lysates were separated in SDS-PAGE and transferred to a PVDF membrane, which were blocked and then probed with primary antibodies overnight at $4{ }^{\circ} \mathrm{C}$ : C/EBP- $\beta$, Pref-1, A-FABP (Santa Cruz), GAPDH (Cell Signaling), $\beta$ actin (Sigma). Oil-Red-O: Lipid content was stained with freshly prepared Oil Red O working solution for 1 hour in formalin fixed cells. 